\title{
Closely spaced dual-wavelength fiber laser using an ultranarrow bandwidth optical filter for low radio frequency generation
}

\author{
H. Ahmad, ${ }^{1, *}$ N. F. Razak, ${ }^{2}$ M. Z. Zulkifli, ${ }^{1}$ F. D. Muhammad, ${ }^{1}$ \\ Y. Munajat, ${ }^{2}$ and S. W. Harun ${ }^{1}$ \\ ${ }^{1}$ Photonics Research Center, Department of Physics, University of Malaya, 50603 Kuala Lumpur, Malaysia \\ ${ }^{2}$ Advanced Photonics Science Institute, Department of Physics, Faculty of Science, \\ University Technology of Malaysia, 81310 Skudai, Johor, Malaysia \\ *Corresponding author: harith@um.edu.my
}

Received 17 March 2014; revised 17 May 2014; accepted 18 May 2014; posted 20 May 2014 (Doc. ID 208341); published 23 June 2014

\begin{abstract}
A dual-wavelength (DW) fiber laser with a closely spaced single longitudinal mode (SLM) output is proposed and demonstrated. The proposed fiber laser utilizes a conventional fiber Bragg grating with a center wavelength of about $1546.8 \mathrm{~nm}$ in conjunction with an ultranarrow bandwidth tunable optical filter to generate the desired DW SLM output. Observations with a very high resolution optical spectrum analyzer, which was capable of achieving resolutions up to $0.16 \mathrm{pm}$, revealed detailed spectral characteristics not characteristically seen before. A channel spacing of up to $58 \mathrm{~nm}$ was realized, and spacing as small as $2 \mathrm{pm}$ was achieved. The minimum channel spacing and its resulting beat frequency are the narrowest observed yet to the best of our knowledge for a DW fiber laser at room temperature. (C2014 Optical Society of America

OCIS codes: (060.0060) Fiber optics and optical communications; (060.2410) Fibers, erbium; (060.2840) Heterodyne; (060.3735) Fiber Bragg gratings; (060.5625) Radio frequency photonics.

http://dx.doi.org/10.1364/AO.53.004123
\end{abstract}

\section{Introduction}

Radio frequency $(\mathrm{RF})$ generation has received great interest in many research fields because of its potential for use in applications, such as communications and satellites, wireless LAN, radio-over-fiber networks, medical devices, and optical sensing components [1-3]. There are two main techniques for generating the RF, and these two techniques include electronic circuitry and optical fiber based schemes. The conventional way used to generate the RF is based on electronic circuitry, however, has some disadvantages in terms of complexity and cost [4]. On the other hand, RF generation based on optical fiber

$1559-128 \mathrm{X} / 14 / 194123-05 \$ 15.00 / 0$

(C) 2014 Optical Society of America schemes is more reliable and practical for $\mathrm{RF}$ applications, which can be realized using various approaches, such as optical-injection locking [5], optical phase-locked loops (OPLL) [6,7], cascaded Brillouin fiber lasers [8], external modulation [9], and dualwavelength $(\overline{\mathrm{DW}})$ laser generation [10-12]. Among these approaches, DW laser generation has the advantage of a simple setup configuration as well as low cost and power consumption. In addition, this approach can also provide tuning ability [12-14] and a narrow linewidth [15], thus making it a desirable approach for $\mathrm{RF}$ generation.

In general, a DW output laser, which is based on different frequencies through beating at the photodetector, has the potential to generate $\mathrm{RF}$, microwave, or even terahertz radiation [16-18]. Various methods have been reported for realizing $R F$ 
generation based on DW fiber lasers, such as by using two-arrayed waveguide gratings (AWGs) [14] implemented with a distributed feedback (DFB) structure and a separate cavity [19], and by using a pair of fiber Bragg gratings (FBGs) [10]. In order to realize $\mathrm{RF}$ generation, an oscillation rate between about $3 \mathrm{kHz}$ and $300 \mathrm{GHz}$ is required.

Narrow DW spacing using various techniques has been reported recently. Liu et al. [20] reported a DW spacing as small as $0.05 \mathrm{~nm}$, which was achieved by applying strain to the polarization-maintaining-FBG (PM-FBG). However, the use of an all-PM linear cavity, which is formed by a uniform PM-FBG and a PM linearly chirped FBG (PM-LCFBG), is expensive and complex. A report from Li et al. [12] shows that by embedding a pair of gratings in phosphate glass fiber, DW spacing as narrow as $0.03 \mathrm{~nm}$ can be obtained. However, the approach of writing FBGs in special fiber would eventually increase the cost of the experiment setup. A different approach was proposed by Xiu-Jie et al. [21], and in this approach one employs a fiber loop mirror (FLP) together with a polarization controller (PC) as a means to generate a fine comblike spectrum. With this technique, a narrow DW spacing of $0.07 \mathrm{~nm}$ can be achieved. Nevertheless, the disadvantage of this method is that the setup is quite complex since a double-stage cascade configuration and an erbium-doped fiber amplifier (EDFA) module are used. In this work, a tunable DW single longitudinal mode (SLM) fiber laser for RF generation is proposed using a regular FBG and an ultranarrow bandwidth (UNB) tunable filter, which can be tuned from $58 \mathrm{pm}$ to as small as $2 \mathrm{pm}$. To the best of our knowledge, this is the narrowest wavelength spacing achieved from DW fiber lasers at room temperature. The resulting $\mathrm{RF}$ generation ranged from 0.25 to $7.27 \mathrm{GHz}$, which also verifies that the dual wavelength is operating in a SLM regime.

\section{Experimental Setup}

The experimental setup of the proposed tunable DW fiber laser is illustrated in Fig. 1. The setup uses a $3 \mathrm{~m}$ long erbium-doped fiber (EDF) with an absorption coefficient of approximately $24 \mathrm{~dB} / \mathrm{m}$ as the active medium of the laser. A $980 \mathrm{~nm}$ laser diode (LD) with a maximum output power of $80 \mathrm{~mW}$ was used to pump the EDF to its excited state, with the pump signal being injected into the cavity via the $980 \mathrm{~nm}$ port of a 980/1550 $\mathrm{nm}$ wavelength division multiplexer (WDM). The $3 \mathrm{~m}$ long EDF is connected at one end to the common port of the WDM, while the other end of the EDF is connected to port 1 of an optical circulator (OC). Port 2 of the OC is connected to an FBG with a center wavelength, $\lambda \mathrm{B}$, of about $1546.8 \mathrm{~nm}$ with a $3 \mathrm{~dB}$ width of $0.019 \mathrm{~nm}$. The FBG acts as a wavelength locker and reflects wavelengths within a range of 1546.781-1546.816 nm back into the cavity to oscillate, while wavelengths that fall outside of this central region are ejected from the cavity. The returning signals travel from port 2 to port 3 of the $\mathrm{OC}$, re-entering the cavity and propagating

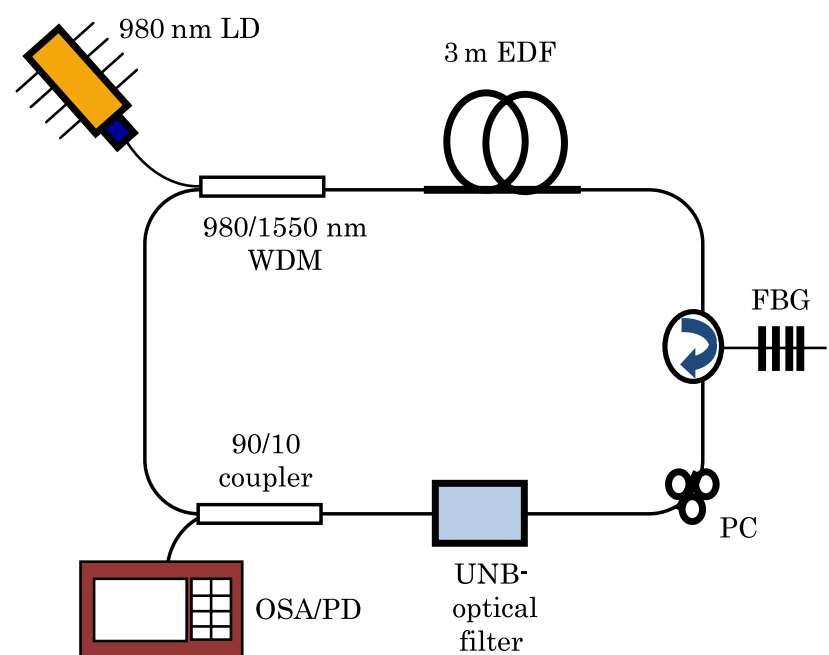

Fig. 1. Schematic diagram of the proposed tunable DW spacing using a tunable filter. $\mathrm{LD}$, laser diode; EDF, erbium-doped fiber; WDM, wavelength division multiplexer; FBG, fiber Bragg grating; OSA/PD, optical spectrum analyzer/potential difference; UNB, ultranarrow bandwidth; PC, polarization controller.

forward toward a PC before reaching the UNB optical filter. The UNB optical filter is a model XTM-50 from Yenista, which has a filter range of $850-50 \mathrm{pm}$, and it is capable of creating both a wide or narrow bandwidth filter.

The filtered output from the UNB optical filter continues to propagate along the laser cavity until it encounters a 90:10 optical coupler, which is used to extract a portion of the beam for further analysis. The remaining $90 \%$ of the optical signal travels back toward the $1550 \mathrm{~nm}$ port of the WDM, thus

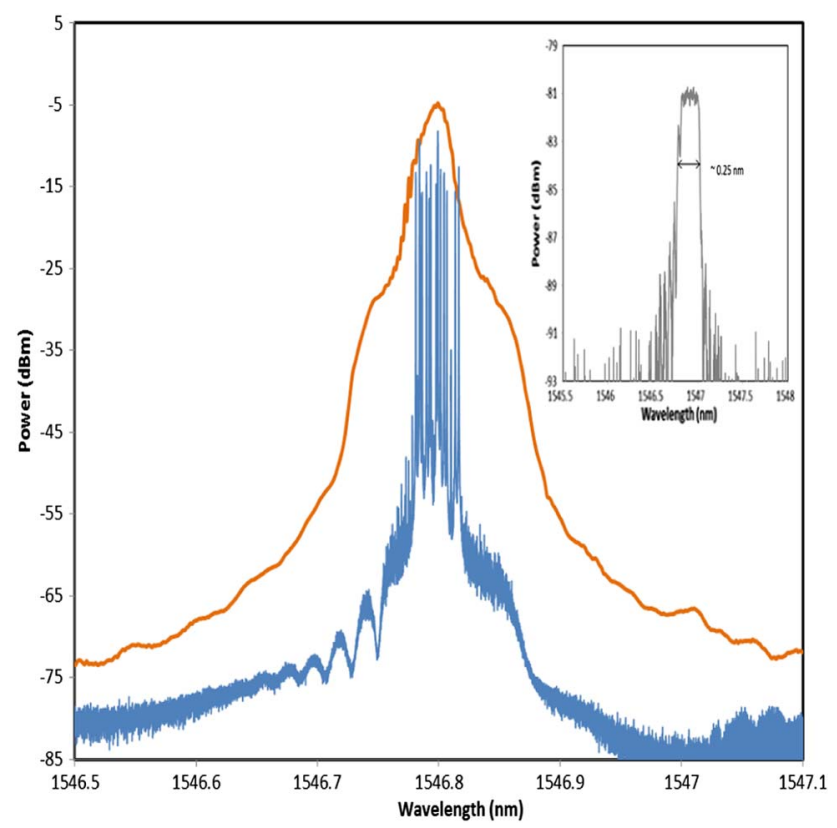

Fig. 2. Enlarged FBG's reflectivity output spectrum observed from $0.02 \mathrm{~nm}$ to $0.16 \mathrm{pm}$ resolutions on an OSA with a $0.5 \mathrm{~nm}$ span (inset: the FBG reflection spectrum observed from a $0.02 \mathrm{~nm}$ resolution on an OSA with a $100 \mathrm{~nm}$ span). 
completing the laser cavity. The total cavity length was measured to be about $15 \mathrm{~m}$. The extracted signal was analyzed by a high resolution APEX AP2051A optical spectrum analyzer (OSA 1), which had a resolution of $0.16 \mathrm{pm}$, and also alternatively with an ANDO AQ6317C optical spectrum analyzer (OSA 2), which had a resolution of $0.02 \mathrm{~nm}$.

\section{Results and Discussion}

The FBG reflection's spectrum is shown as an inset in Fig. 2, which was taken using an OSA with a resolution of $0.02 \mathrm{~nm}$. The spectrum has a full width at half-maximum (FWHM) of $0.25 \mathrm{~nm}$. This FBG, which is connected to the ring cavity through the OC, allows lasing action with the output shown in Fig. 2. The outer trace was taken using a standard OSA 2 with a resolution of $0.02 \mathrm{~nm}$, and the inner trace was taken using an OSA 1 with a resolution of $0.16 \mathrm{pm}$. As can be seen from the figure, the trace taken with the high resolution OSA provides details of the lasing trace, which was composed of many lines that are indicative of many longitudinal modes oscillating at the same time. The wavelength span was from 1546.76 to $1546.82 \mathrm{~nm}$, and the peak power ranged from -8 to $-16.97 \mathrm{dBm}$.

For the DW generation, these many longitudinal modes have to be reduced to only two modes of oscillation. This step was aided by use of the UNB optical filter, as shown in Fig. 1. The bandwidth of the UNB is adjustable so that it can be varied from 2.0 to $58.0 \mathrm{pm}$.

The measured trace using the UNB optical filter in the cavity is shown in Fig. 3(a), which was taken from OSA 2 with a linewidth of $\overline{0.019} \mathrm{~nm}$ at $3 \mathrm{~dB}$. The trace

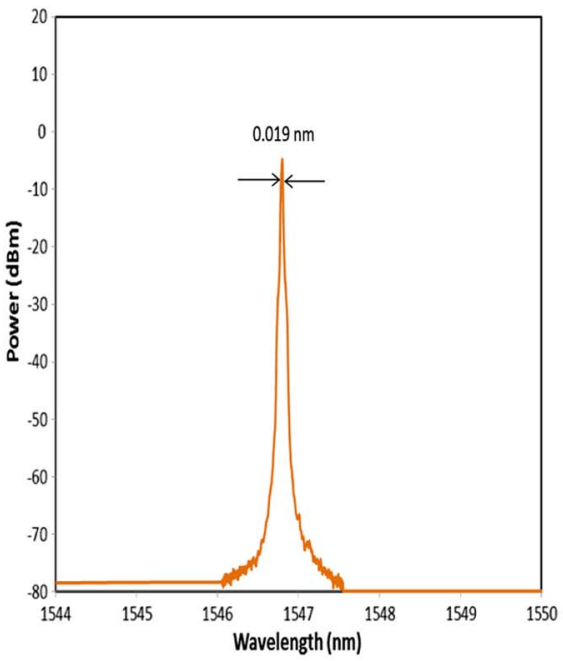

(a)

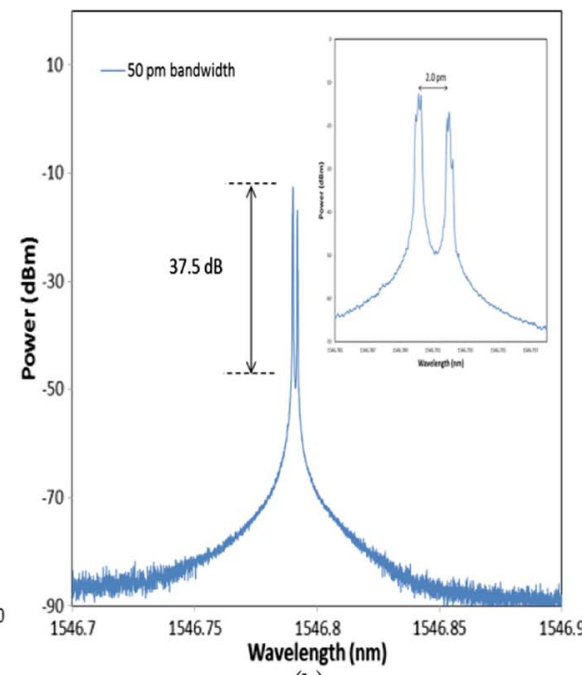

(b)

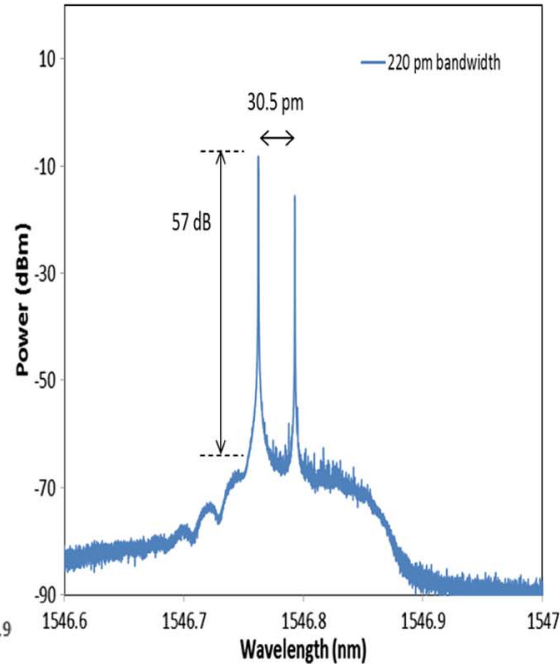

(c)

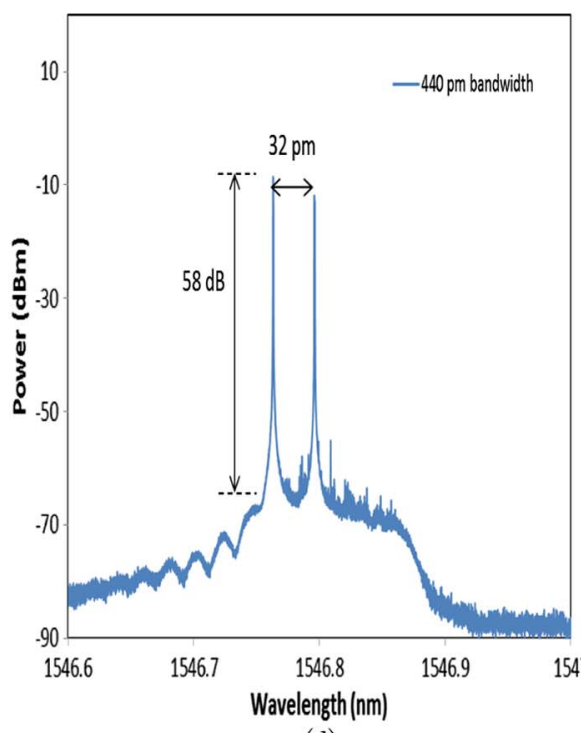

(d)

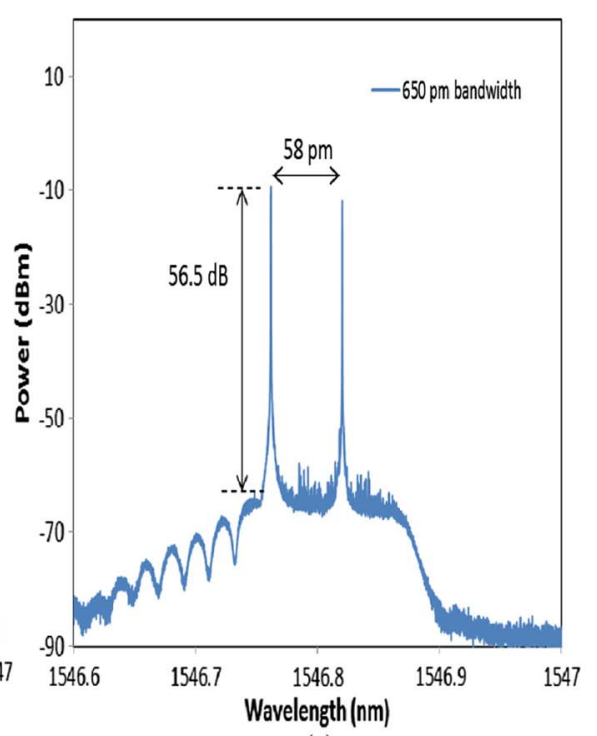

(e)

Fig. 3. DW fiber laser output with different spacing. (a) Output spectrum taken from OSA 2 with a resolution of $0.02 \mathrm{~nm}$, (b) output spectrum taken from OSA 1 with a resolution of $0.16 \mathrm{pm}$ and giving a DW spacing of $2 \mathrm{pm}$ with a signal-to-noise ratio (SNR) of $37.5 \mathrm{~dB}$, (c) spectrum from OSA 1 giving a DW spacing of $30.5 \mathrm{pm}$ with a SNR of $57 \mathrm{~dB}$, (d) spectrum from OSA 1 giving a DW spacing of $32.5 \mathrm{pm}$ with a SNR of $58 \mathrm{~dB}$, and (e) spectrum from OSA 1 giving a DW spacing of $58.0 \mathrm{pm}$ with a SNR of 56.5. 
shows a single line oscillation riding on top of a broad amplified spontaneous emission (ASE) output. Detailed analysis of this output was taken using OSA 1, as shown in Fig. 3(b), with a resolution of $0.16 \mathrm{pm}$. It can be inferred from this data that there were two lines oscillating simultaneously, and these two lines had linewidths of 0.22 and $0.02 \mathrm{pm}$ at $3 \mathrm{~dB}$ with a line spacing of $2 \mathrm{pm}$. At this high resolution, OSA 1 does help to provide a deeper analysis of the laser oscillation with wavelength spacing as indicated above. Using an FBG together with a UNB optical filter, we were thus able to create a DW output as shown. By adjusting the bandwidth of the UNB optical filter from 50 to $220 \mathrm{pm}$, the spacing between the two lines can be further increased to $30.5 \mathrm{pm}$, as shown in Fig. 3(c). By further adjusting the setting of the bandwidth filter, the spacing was further increased from 32 to $58 \mathrm{pm}$ at settings of 440 and 650 pm, respectively, as shown in Figs. 3(d) and 3(e).

Figure 4 shows the measured RF spectrum using a high-speed photodetector (Agilent $83440 \mathrm{C}$, with a bandwidth of $20 \mathrm{GHz}$ ) together with an RF spectrum analyzer (Anritsu MS2667C RFSA); these were taken from the DW output of the ring laser. From the trace, for the case where the spacing of the two lines was at $2 \mathrm{pm}$, the measured $\mathrm{RF}$ was $0.251 \mathrm{GHz}$. Due to the presence of a single line in the RF spectrum analyzer, it can be inferred that the DW fiber laser was operating in a SLM regime.

Figure 5 shows the measured RF spectrum output that was taken at different DW spacings of $2,30.5$, 32 , and $58 \mathrm{pm}$, which gave RF signals at 0.251 , $3.82,4.07$, and $7.27 \mathrm{GHz}$, respectively. It should be noted that the spectrum given in Fig. $\underline{5}$ is a superposition of the different frequency peaks in the actual measurement; only a single peak is visible for each channel spacing configuration.

The above measurement clearly indicates that the proposed configuration provides a wide tunability in the generation of the RF signal. This will find

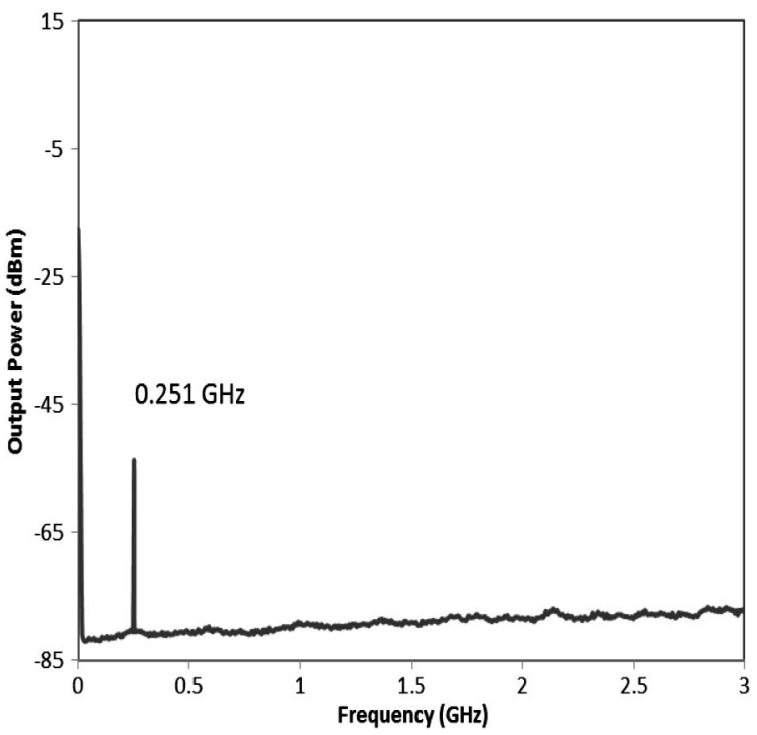

Fig. 4. RF beat spectrum at a DW spacing of $2 \mathrm{pm}$.

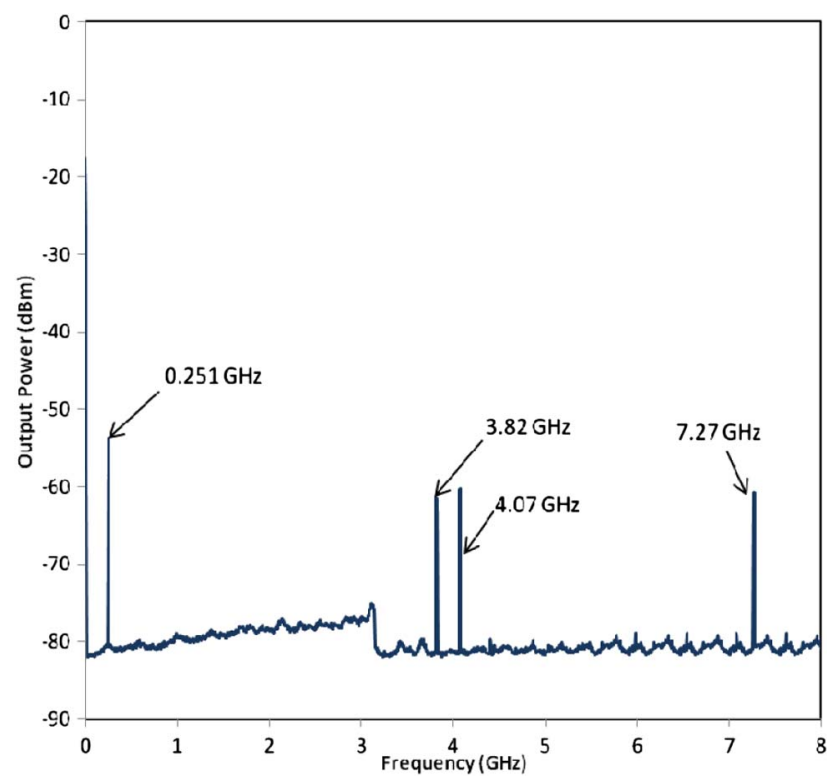

Fig. 5. RF beat spectrum with respect to each different wavelength spacing as shown in Figures $\underline{3(\mathrm{~b})}-\underline{3(\mathrm{e})}$.

applications in optical sensing and as an RF source for radio-over-fiber networks.

\section{Conclusion}

A tunable DW SLM fiber laser using a regular FBG together with an ultranarrow tunable filter in a ring cavity is demonstrated. Spectrum measurements were taken using a high resolution OSA with a resolution of $0.16 \mathrm{pm}$, which allowed for an in-depth analysis of the optical output that could not be realized before. The proposed design allows for a tunability of up to $58 \mathrm{pm}$ between the two generated lasing wavelengths, which then gives it a corresponding tunability range in the $R F$ spectrum. The minimum channel spacing of $2 \mathrm{pm}$ is to our knowledge the narrowest channel spacing reported yet, with a corresponding beat signal of $0.251 \mathrm{GHz}$. Observations of the RF output confirmed SLM operation with only a single peak. The proposed laser has significant potential for use as RF sources for radio-over-fiber applications.

We would like to thank University of Malaya for providing the funding for this work under grant RU002/2013.

\section{References}

1. J. Yao, "Microwave photonics," J. Lightwave Technol. 27, 314-335 (2009).

2. A. Rosen, M. A. Stuchly, and A. V. Vorst, "Applications of RF/ microwaves in medicine," IEEE Trans. Microwave Theor. Tech. 50, 963-974 (2002).

3. N. J. Gomes, M. Morant, A. Alphones, B. Cabon, J. E. Mitchell, C. Lethien, and S. Iezekiel, "Radio-over-fiber transport for the support of wireless broadband services," J. Opt. Netw. 8, 156-178 (2009).

4. M. Feng, S. C. Shen, D. C. Caruth, and A. J. Huang, "Device technologies for RF front-end circuits in nextgeneration wireless communications," Proc. IEEE 92, 354-375 (2004) 
5. P. Bouyer, T. L. Gustavson, K. G. Haritos, and M. A. Kasevich, "Dual-frequency Brillouin fiber laser for optical generation of tunable low-noise radio frequency/microwave frequency," Opt. Lett. 21, 1502-1504 (1996).

6. H. R. Rideout, J. S. Seregelyi, S. Paquet, and J. Yao, "Discriminator-aided optical phase-lock loop incorporating a frequency down-conversion module," IEEE Photon. Technol. Lett. 18, 2344-2346 (2006).

7. U. Gliese, T. N. Nielsen, M. Bruun, E. Lintz Christensen, K. E. Stubkjzr, S. Lindgren, and B. Broberg, "A wideband heterodyne optical phase-locked loop for generation of $3-18 \mathrm{GHz}$ microwave carriers," IEEE Photon. Technol. Lett. 4, 16-18 (2008).

8. J. Liu, L. Zhan, P. Xiao, Q. Shen, G. Wang, Z. Wu, X. Liu, and L. Zhang, "Optical generation of tunable microwave signal using cascaded Brillouin fiber laser," IEEE Photon. Technol. Lett. 24, 22-24 (2012).

9. G. H. Qi, J. P. Yao, J. Seregelyi, S. Paquet, and C. Bélisle, "Generation and distribution of a wideband continuously tunable millimeter-wave signal with an optical external modulation technique," IEEE Trans. Microwave Theor. Tech. 53, 30903097 (2005).

10. X. Chen, Z. Deng, and J. Yao, "Photonic generation of microwave signal using a dual-wavelength single-longitudinalmode fiber ring laser," IEEE Trans. Microwave Theor. Tech. 54, 804-809 (2006).

11. A. W. Al-Alimi, M. H. Al-Mansoori, A. F. Abas, M. A. Mahdi, F. R. M. Adikan, and M. Ajiya, "A stabilized tunable dual wavelength erbium-doped fiber laser with equal output power," Laser Phys. 19, 1850-1853 (2009).

12. L. Li, A. Schülzgen, X. Zhu, J. V. Moloney, J. Albert, and N. Peyghambarian, "1 W tunable dual-wavelength emission from cascaded distributed feedback fiber lasers," Appl. Phys. Lett. 92, 051111 (2008).

13. H. Ahmad, A. A. Latif, M. I. M. A. Khudus, A. Z. Zulkifli, M. Z. Zulkifli, K. Thambiratnam, and S. W. Harun, "Highly stable graphene-assisted tunable dual-wavelength erbium-doped fiber laser," Appl. Opt. 52, 818-823 (2013).

14. H. Ahmad, A. A. Latif, J. M. Talib, and S. W. Harun, "Tunable, low frequency microwave generation from AWG based closelyspaced dual-wavelength single-longitudinal-mode fibre laser," J. Eur. Opt. Soc. 8, 1-5 (2013).

15. H. Zhu, C. Tu, T. Lei, W. Guo, Y. Li, F. Lu, X. Dong, and D. Wei, "Dual-wavelength narrow-linewidth light source with ultranarrow wavelength spacing based on the pump-induced thermal effects in an Er-Yb-codoped distributed-Bragg-reflector fiber laser," Opt. Eng. 47, 094301 (2008).

16. Y. Geng, X. Tan, X. Li, and J. Yao, "Compact and widely tunable terahertz source based on a dual-wavelength intracavity optical parametric oscillation,” Appl. Phys. B 99, 181-185 (2010).

17. T. Taniuchi, J. Shikata, and H. Ito, "Tunable terahertz-wave generation in DAST crystal with dual-wavelength KTP optical parametric oscillator," Electron. Lett. 36, 1414-1416 (2000).

18. M. Alouini, M. Brunel, F. Bretenaker, M. Vallet, and A. Le Floch," Dual tunable wavelength Er, Yb:glass laser for terahertz beat frequency generation," IEEE Photon. Technol. Lett. 10, 1554-1556 (1998).

19. J. Sun, Y. Dai, X. Chen, Y. Zhang, and S. Xie, "Stable dualwavelength DFB fiber laser with separate resonant cavities and its application in tunable microwave generation," IEEE Photon. Technol. Lett. 18, 2587-2589 (2006).

20. D. Liu, N. Q. Ngo, X. Y. Dong, S. C. Tjin, and P. Shum, "A stable dual-wavelength fiber laser with tunable wavelength spacing using a polarization-maintaining linear cavity," Appl. Phys. B 81, 807-811 (2005).

21. J. Xiu-Jie, L. Yan-Ge, S. Li-Bin, G. Zhan-Cheng, F. Sheng-Gui, L. Feng-Nian, Y. Shu-Zhong, and D. Xiao-Yi, "Realization of stable narrow linewidth dual-wavelength lasing in an erbiumdoped fibre laser by cleaving the wavelength-selective filter spectrum," Chin. Phys. Lett 23, 2092-2094 (2006). 